\title{
Tunable Single-Longitudinal-Mode High-Power Fiber Laser
}

\author{
Jonas K. Valiunas and Gautam Das \\ Department of Physics, Lakehead University, 955 Oliver Road, Thunder Bay, ON, Canada P7B 5E1 \\ Correspondence should be addressed to Gautam Das, gdas@lakeheadu.ca
}

Received 1 October 2011; Accepted 5 December 2011

Academic Editor: Frédéric Smektala

Copyright ( $\odot 2012$ J. K. Valiunas and G. Das. This is an open access article distributed under the Creative Commons Attribution License, which permits unrestricted use, distribution, and reproduction in any medium, provided the original work is properly cited.

\begin{abstract}
We report a novel CW tunable high-power single-longitudinal-mode fiber laser with a linewidth of $\sim 9 \mathrm{MHz}$. A tunable fiber Bragg grating provided wavelength selection over a $10 \mathrm{~nm}$ range. An all-fiber Fabry-Perot filter was used to increase the longitudinal mode spacing of the laser cavity. An unpumped polarization-maintaining erbium-doped fiber was used inside the cavity to eliminate mode hopping and increase stability. A maximum output power of $300 \mathrm{~mW}$ was produced while maintaining single-longitudinalmode operation.
\end{abstract}

\section{Introduction}

Fiber lasers are established as robust and reliable devices with a variety of applications in industry and medicine due to their unique characteristics, such as all-fiber designs, compact size, cost-effective production and operation, and the no need for realignment or external cooling. High-power single-wavelength and multiwavelength infrared fiber lasers are very attractive for applications in optical communications, sensing, spectroscopy, biomedical instrumentation, and nonlinear optics. The continued progress in fiber pumping techniques, advanced fiber designs, and fabrication processes, as well as the availability of high-power pump diodes, has assisted in the development of high-power fiber lasers [1-5]. Fiber lasers have found applications in temperature and strain sensors [6-11], medical diagnostics [12-14], and industrial processing [15]. High-power fiber lasers using erbium-ytterbium codoped fibers as the gain medium, which operates in the eye-safe $(1.5 \mu \mathrm{m}$ to $1.6 \mu \mathrm{m})$ spectral range, can now compete with traditional solid-state bulk lasers. The applications of recently reported singlewavelength [16, 17] and multiwavelength [18, 19] highpower fiber lasers were limited due to large linewidth of the lasing wavelength, multi-longitudinal-mode oscillations, small tuning range, and complex designs.

In this paper we present a novel tunable, high-power, single-wavelength, single-longitudinal-mode, fiber ring laser.

\section{Experimental Setup}

The experimental setup of the fiber laser is shown in Figure 1. The resonant cavity consists of a high-power polarizationindependent optical isolator (OI), which guaranteed the unidirectional propagation and thus eliminated the spatial hole-burning effects [20]; an all-fiber polarization controller; a commercially available tunable fiber Bragg grating (TFBG) with a tuning range of $10 \mathrm{~nm}(1565 \mathrm{~nm}-1575 \mathrm{~nm})$; a $4 \mathrm{~m}$ long double-clad erbium-ytterbium codoped (DC-EYDF) fiber with core/cladding diameters of $10 / 131 \mu \mathrm{m}$ which was used as the gain medium. In general to produce high output power from a ring laser, a longer length of the active medium is required which leads to a smaller longitudinal-mode spacing and a narrower laser linewidth. A double-clad erbiumytterbium codoped fiber (DC-EYDF), with high conversion efficiency, as an active medium eliminated the requirement for a long length of the active medium. The DC-EYDF (CorActive) had much greater absorption and coupling efficiency compared to that of a circularly symmetric doubleclad fiber due to its hexagonal inner cladding [21]. An unpumped polarization-maintaining erbium-doped fiber (PMEDF) was used as a saturable absorber (SA) inside the cavity to reduce the mode hopping of the lasing wavelength. The SA had an elliptical core with dimensions, peak absorption, cutoff wavelength, and a numerical aperture of $3.8 \times 14.8 \mu \mathrm{m}$, $10.8 \mathrm{~dB} / \mathrm{m}$ at $1535 \mathrm{~nm}, 1371 \mathrm{~nm}$, and 0.15 , respectively. To 


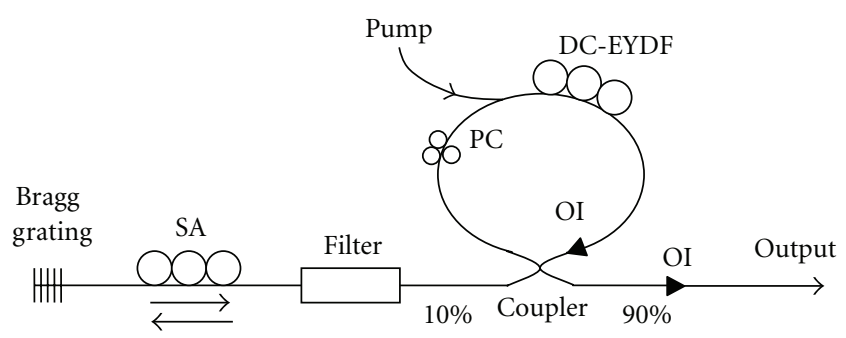

FIGURE 1: Experimental setup of our fiber laser.



Figure 2: All-fiber Fabry-Perot filter [22].

increase the effective longitudinal-mode spacing and stability of the laser wavelength, an all-fiber Fabry-Perot filter with wide free spectral range (FSR) was used within the cavity. The Fabry-Perot filter was formed by splicing two $2 \times 2$ single-mode fused fiber couplers (split ratio 99:1) together (Figure 2), which helped to eliminate the mode hopping of the laser wavelength by increasing the effective longitudinalmode spacing of the laser cavity [22]. In general the length of the ring cavity is longer than the FP filter cavity; thus, the presence of the filter inside the cavity produces the vernier effect and increases the longitudinal-mode spacing of the cavity [23-25]. In our experiment the length of the Fabry-Perot filter was $\sim 0.4 \mathrm{~m}$, which corresponds to a free spectral range of $\mathrm{FSR}_{\mathrm{FP}} \approx 514 \mathrm{MHz}$. A multimode laser diode at $976 \mathrm{~nm}$ with a maximum output power of $10 \mathrm{~W}$ was used to pump the laser cavity. A multimode fused fiber coupler $(6 \times 1$ multimode pump power combiner $)$ was used as a $976 / 1550 \mathrm{~nm}$ wavelength division multiplexing coupler to couple power from the pump laser into the DCEYDF fiber. The fiber Bragg grating, saturable absorber, Fabry-Perot filter, and the ring together formed an overlapping cavity configuration, where the output of the ring resonator was modulated by the output of the Fabry-Perot cavity. The output of the laser was obtained from the $90 \%$ port of a 90:10 fused fiber coupler. A polarizationindependent optical isolator was used to reduce any back reflection from the output port. The output of the laser was monitored using an optical spectrum analyzer with a resolution of $1.25 \mathrm{GHz}$, a scanning Fabry-Perot spectrum analyzer (SFPSA) of resolution $6.7 \mathrm{MHz}$ and a power meter.

\section{Results and Discussions}

Figure 3 shows the tunable characteristics of the laser for an input pump power of $\sim 700 \mathrm{~mW}$ at a wavelength of $976 \mathrm{~nm}$.

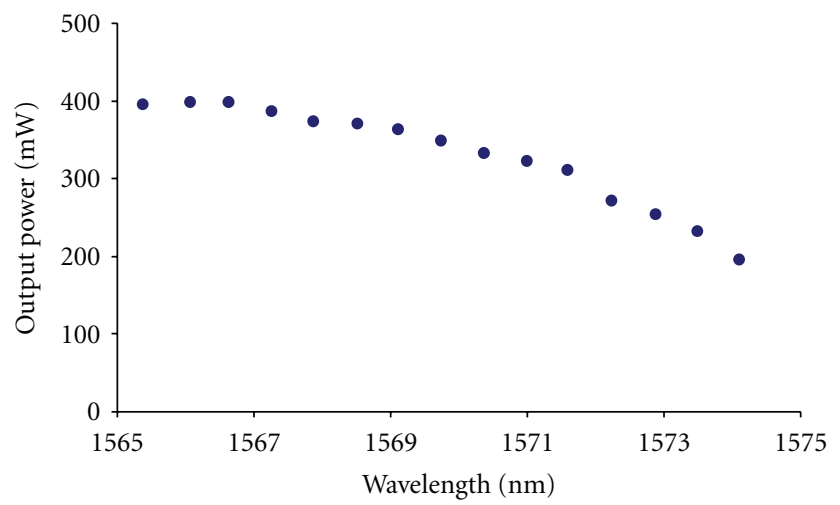

Figure 3: Output power of the laser at $\sim 0.6 \mathrm{~nm}$ intervals from $1565 \mathrm{~nm}$ to $1575 \mathrm{~nm}$.

The laser was tunable from $1565 \mathrm{~nm}$ to $1575 \mathrm{~nm}$, which was limited by the tunability of the grating, and the minimum tunable interval was $0.01 \mathrm{~nm}$. The nonuniform output power over the wavelength range is due to the erbium-ytterbium codoped fiber emission, which decreases in this region.

Figure 4(a) shows the output of the laser obtained by the OSA without any saturable absorber inside the cavity, and Figure 4(b) is the input-output characteristics of the laser. The maximum power obtained from the laser was $\sim 600 \mathrm{~mW}$ for a pump power of $\sim 1300 \mathrm{~mW}$ at $\lambda=1565.52 \mathrm{~nm}$. The threshold pump power and efficiency of the laser were $\sim 200 \mathrm{~mW}$ and $\sim 49 \%$, respectively. The effective length of our ring cavity and the length of the FP filter were $\sim 9 \mathrm{~m}$ and $\sim 0.4 \mathrm{~m}$, respectively. The corresponding FSR of the ring cavity was $\sim 24 \mathrm{MHz}$ and of the Fabry-Perot filter was $\sim 514 \mathrm{MHz}$. The theoretical resonance linewidth (FWHM) of the FP filter was $2 \mathrm{MHz}$. The presence of this filter resulted in an effective longitudinal-mode spacing of $514 \mathrm{MHz}$ for the laser cavity.

To study the longitudinal-mode structure of the laser, a scanning Fabry-Perot spectrum analyzer $(F S R=2 \mathrm{GHz}$ ) of resolution $6.7 \mathrm{MHz}$ and NuView software developed by EXFO was used. The maximum output power with singlelongitudinal-mode oscillation was less than $50 \mathrm{~mW}$ with a $4 \mathrm{~m}$ DC-EYDF. The experiment was repeated using $1 \mathrm{~m}$ of DC-EYDF fiber to reduce the effective cavity length to $6 \mathrm{~m}$, which corresponds to longitudinal-mode spacing of $34 \mathrm{MHz}$. This allowed us to maintain a lasing wavelength that was single-longitudinal-mode and free from mode hopping to a maximum output of $100 \mathrm{~mW}$. Figure 5 was obtained using the scanning Fabry-Perot spectrum analyzer (SFPSA) and confirmed the single-longitudinal-mode oscillation of the laser. At high output powers, the laser suffered from mode hopping and oscillations of multiple longitudinal modes.

In order to improve the stability of the laser output at high power, we incorporated an unpumped polarization maintaining erbium-doped fiber as a saturable absorber into the cavity. A series of experiments were carried out using $1 \mathrm{~m}, 3 \mathrm{~m}$, and $5 \mathrm{~m}$ of PM-EDF to find the optimum length of the SA, which would increase the stability of the lasing wavelength, by reducing mode hopping, and without 


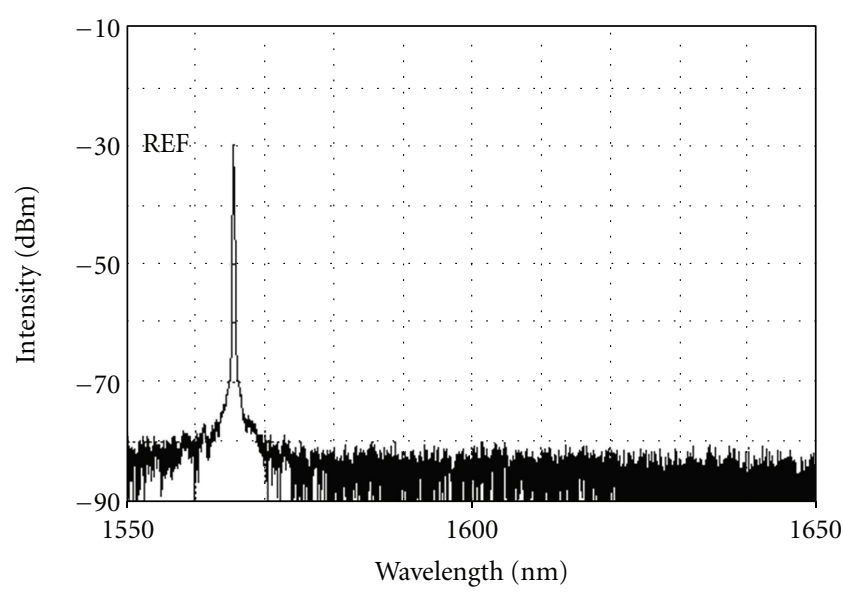

(a)

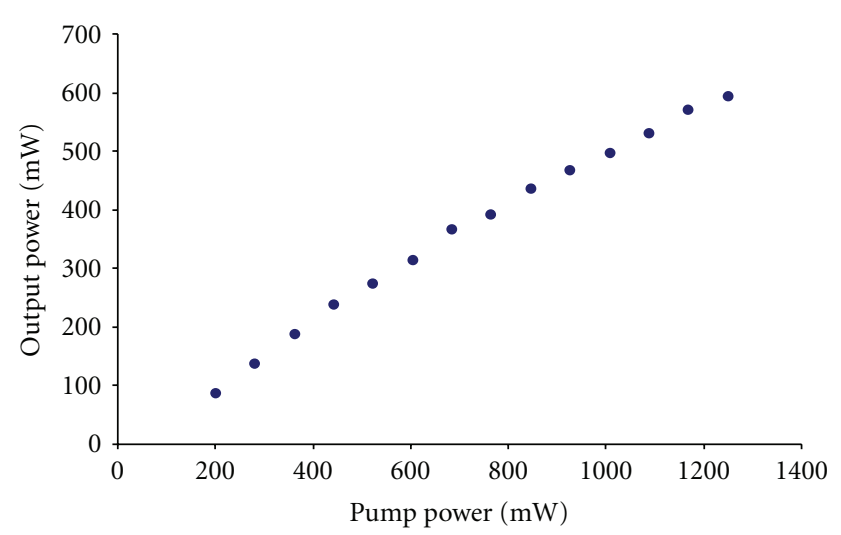

(b)

FIGURE 4: (a) Output of the laser obtained with OSA; (b) input-output characteristics of the laser without the SA for $\lambda=1565.52 \mathrm{~nm}$.

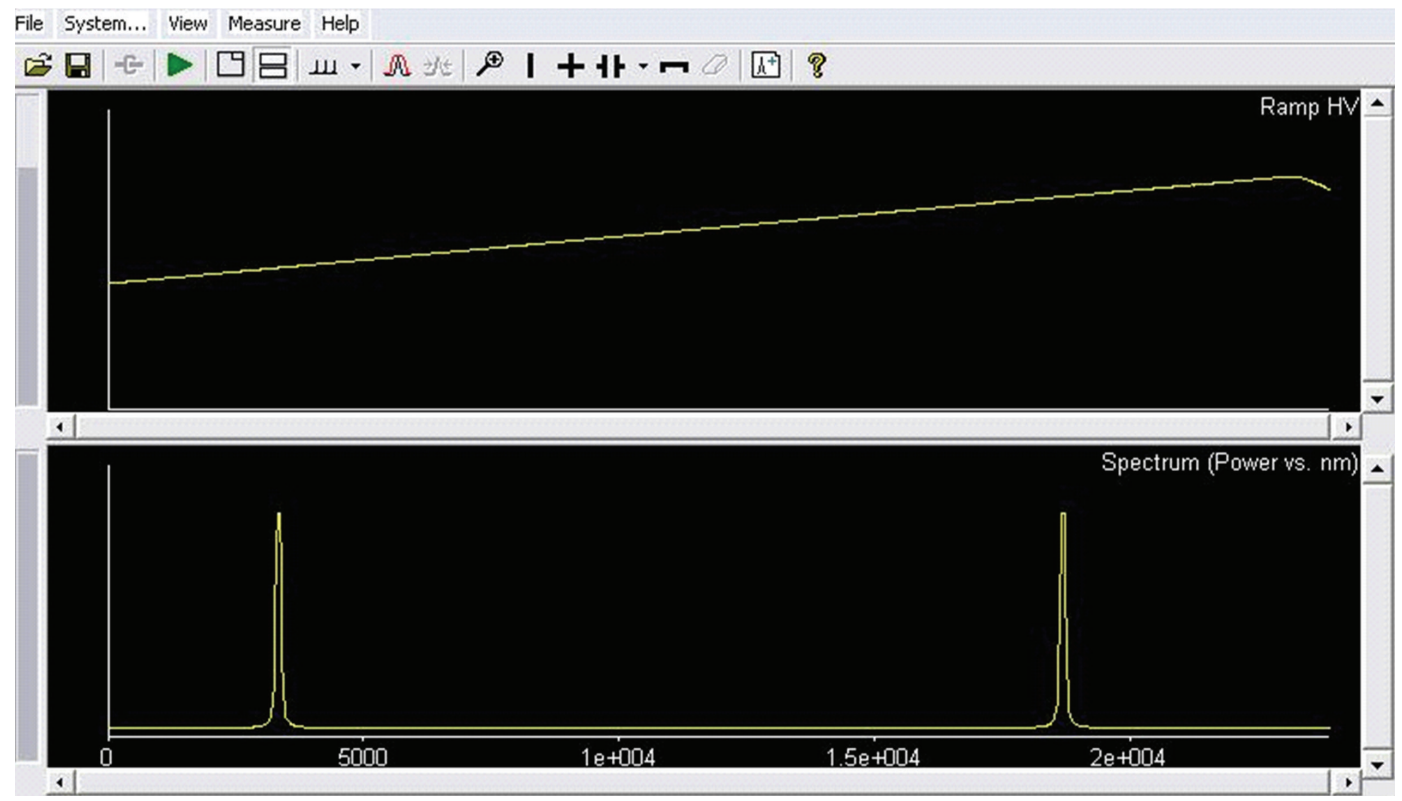

Figure 5: Output of the laser obtained using scanning Fabry-Perot spectrum analyzer.

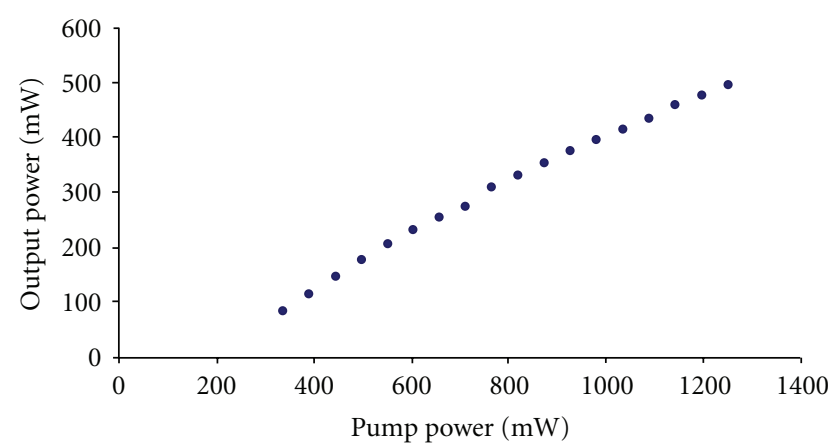

FIGURE 6: Input-output characteristics of the laser with $5 \mathrm{~m}$ of SA inside the cavity at $\lambda=1565.52 \mathrm{~nm}$.

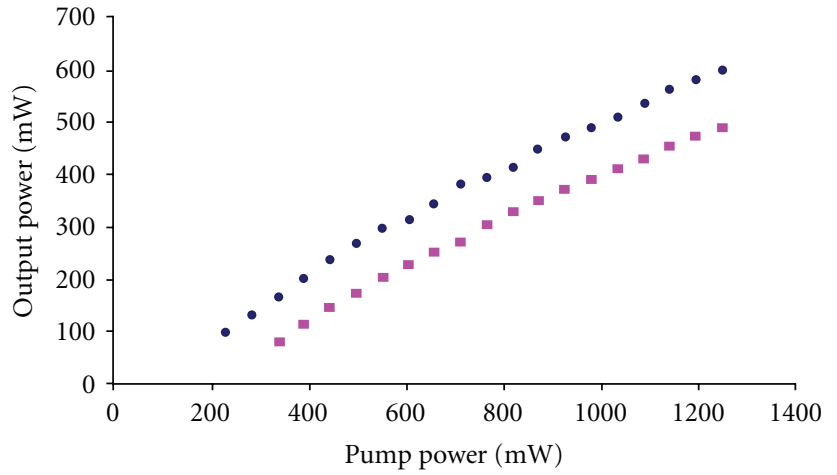

- Without SA

- With $5 \mathrm{~m} \mathrm{SA}$

FIGURE 7: Input-output characteristics of the laser with and without the SA. 


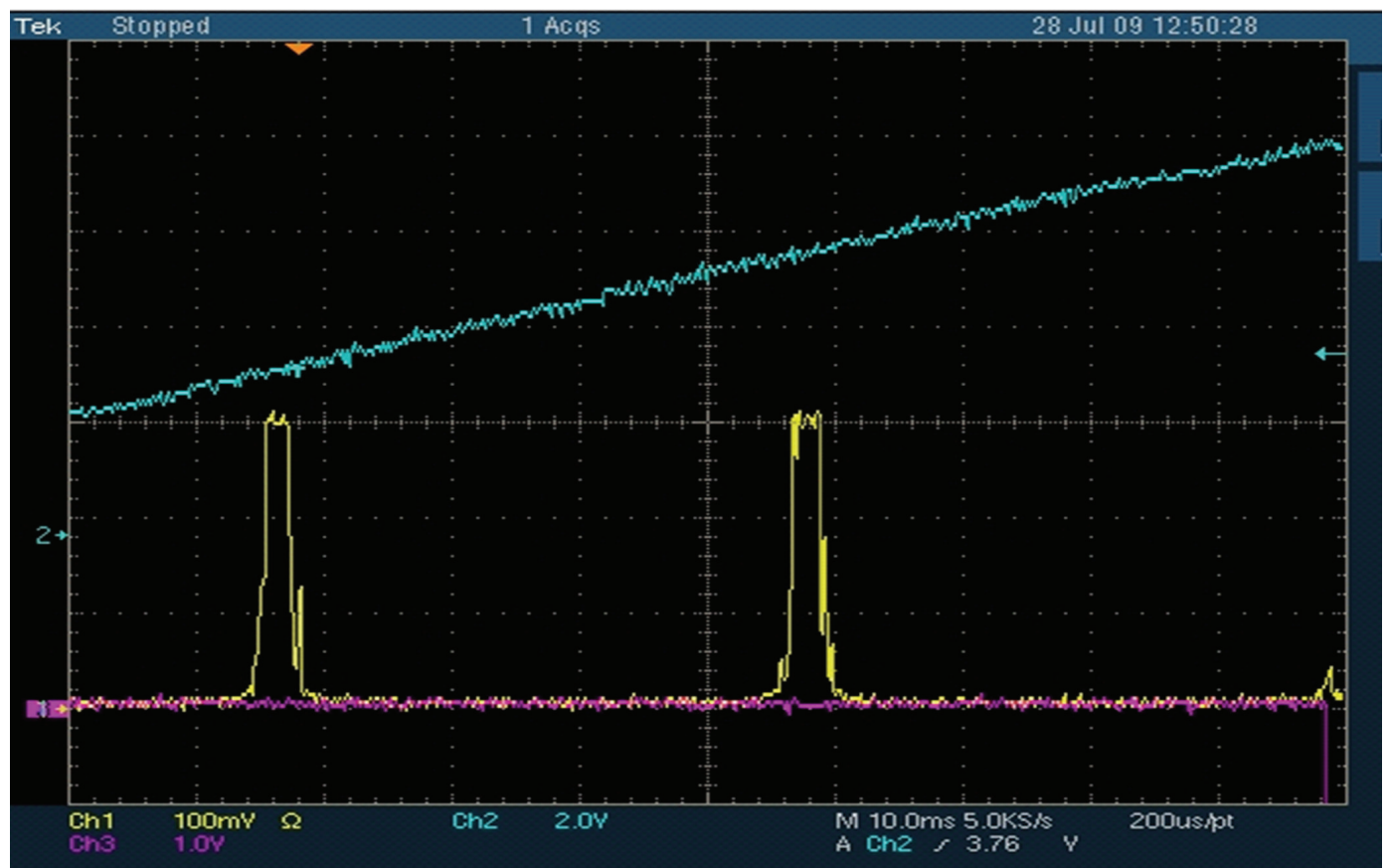

FIGURE 8: Output of the laser obtained using SFPSA when the laser operated in the pulsed regime.

largely attenuating the laser output power. We found that the $5 \mathrm{~m}$ PM-EDF provided the stability needed while still allowing a high output power. Figure 6 shows the inputoutput characteristics of the laser with a SA inside the cavity. The polarization controller and polarization-maintaining EDF helped to produce and maintain quasilinearly polarized light inside the saturable absorber and thus increased the stability of the transient grating. The maximum output power achieved while maintaining single-longitudinal-mode operations was more than $300 \mathrm{~mW}$ with the SA.

The counter-propagating light waves inside the saturable absorber formed a transient grating with a reflection bandwidth of $20 \mathrm{MHz}$. The transient grating acted as a tracking filter, where the central frequency tracked the lasing mode and thus eliminated mode hopping. The transient grating was capable of adjusting itself, within a few milliseconds, to any sudden changes in the laser cavity, such as changes in temperature or other environmental fluctuations [26]. The effective cavity length of the laser was $\sim 19 \mathrm{~m}$, which corresponds to a longitudinal-mode spacing of $\sim 10 \mathrm{MHz}$. Though the longer cavity length decreased the longitudinalmode spacing of the cavity, the presence of the FabryPerot filter increased the longitudinal-mode spacing to 514 $\mathrm{MHz}$.

Figure 7 shows the input-output characteristics of the laser with and without the SA, which shows the effect of SA on the output of the laser. The laser was oscillating in single-longitudinal-mode when more than $300 \mathrm{~mW}$ of output power was produced.

The 3-dB linewidth of the laser at lower power $(\sim 100 \mathrm{~mW})$ was $\sim 8.75 \mathrm{MHz}$ as measured by the SFPSA and NuView software. The linewidth of the laser at high pump power was larger when compared to the theoretical value based on the Schawlow-Townes formula [27]. We found that the linewidth of the laser increased with increasing pump power. It was reported that the wider linewidth in the erbium-ytterbium-co-doped fiber laser is due to the temperature fluctuations induced by the pump intensity noise inside the core of the fiber or due to four-wave mixing between various longitudinal modes in the laser cavities [2831].

We found that it was possible to maintain the relative phases of the modes by adjusting the polarization controller plates inside the cavity when the laser was oscillating in multiple longitudinal modes. This phenomenon is referred to as passive mode locking and produced periodic pulses at the output of the laser. Figure 8 shows the output of the laser at high power obtained from the scanning Fabry-Perot spectrum analyzer, after achieving mode locking through adjustments of the polarization controller plates inside the cavity. At the optimum location of the polarization controller plates all the modes collapsed into a single pulse with a large bandwidth $(\sim 60 \mathrm{MHz})$. This result was due to the presence of the saturable absorber inside the cavity and is known as passive mode locking. We also found that even without the presence of the saturable absorber the laser produces pulses at high powers when the polarization controller plates were adjusted properly. This is another type of passive mode locking known as nonlinear polarization rotation mode locking.

Figure 9 is the output of the laser when operated at high power and was obtained with a high-speed photo detector $(2.5 \mathrm{GHz})$ and an oscilloscope with a $1 \mathrm{GHz}$ bandwidth. Thus, we could operate the laser in either CW or pulsed mode by adjusting the polarization controller plates inside the cavity. 


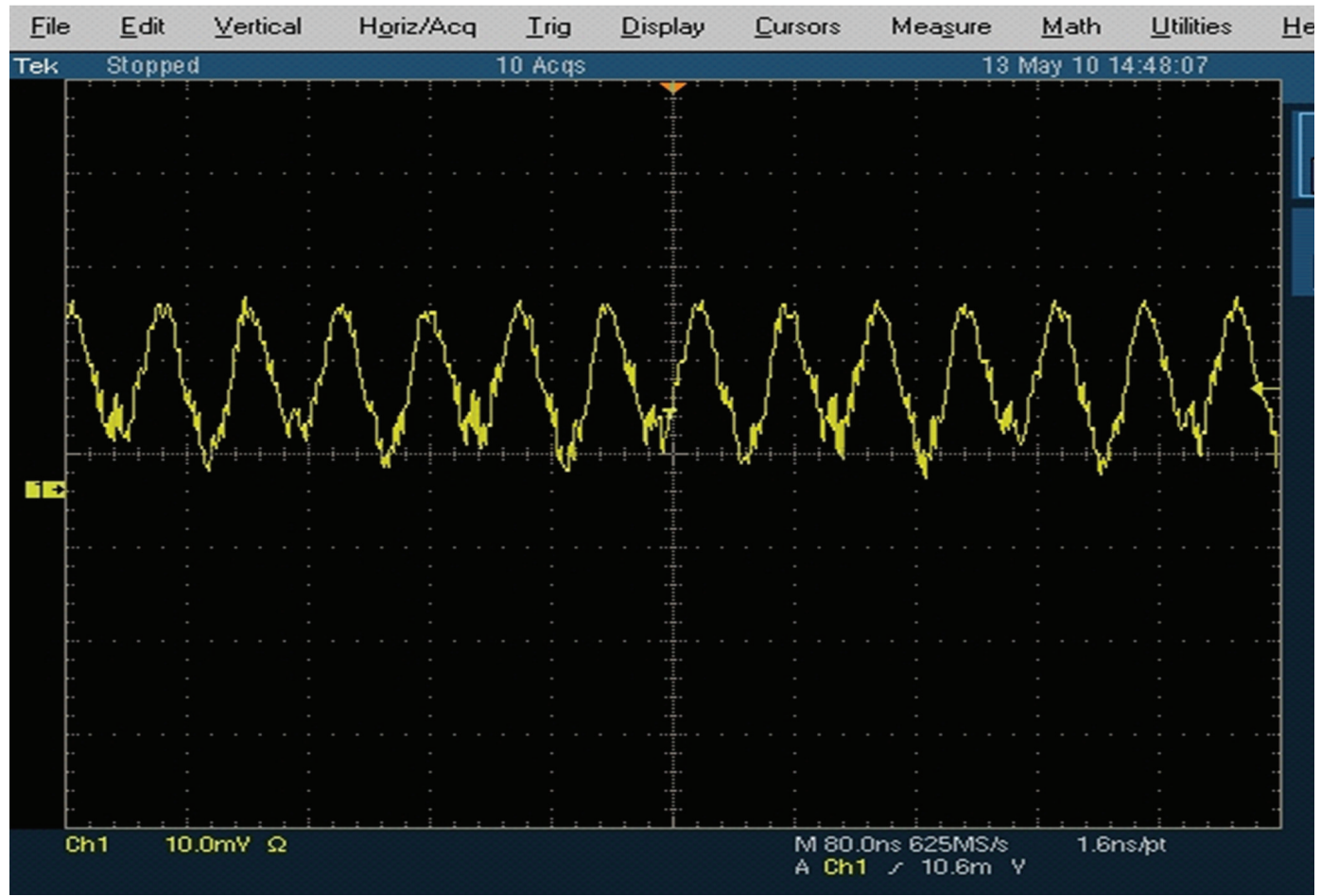

Figure 9: Output of the laser obtained from the oscilloscope.

\section{Conclusion}

We have demonstrated a high-power single-wavelength, single-longitudinal-mode fiber laser. The laser was free from mode hopping to a maximum output power of $\sim 300 \mathrm{~mW}$. The laser was tunable over $1565 \mathrm{~nm}$ to $1575 \mathrm{~nm}$ and had a linewidth of $\sim 9 \mathrm{MHz}$. The presence of SA inside the cavity increased the stability of the laser. The laser could be operated in the CW and pulsed mode through manipulation of the polarization controller. Due to the narrow linewidth and high output power, this laser could find applications in developing sensor based on nonlinear effects such as stimulated Brillouin scattering.

\section{References}

[1] J. Nilsson, J. K. Sahu, Y. Jeong et al., "High power fiber lasers: new developments," in Proceedings of the SPIE: Advances in Fiber Devices, vol. 4974, pp. 50-59, January 2003.

[2] J. K. Sahu, Y. Jeong, C. Codemard et al., "Tunable narrow linewidth high power erbium: ytterbium co-doped fiber laser," in Proceedings of the Conference on Lasers and Electro-Optics (CLEO '04), vol. 1, pp. 159-160, May 2004.

[3] M. Laroche, P. Jander, W. A. Clarkson, J. K. Sahu, J. Nilsson, and Y. Jeong, "High power cladding-pumped tunable Er, Ybdoped fibre laser," Electronics Letters, vol. 40, no. 14, pp. 855856, 2004.

[4] Y. Jeong, J. K. Sahu, D. N. Payne, and J. Nilsson, “ytterbiumdoped large-core fiber laser with $1.36 \mathrm{~kW}$ continuous-wave output power," Optics Express, vol. 12, no. 25, pp. 6088-6092, 2004.
[5] Y. Jeong, J. Nilsson, J. K. Sahu et al., "Single-mode planepolarized ytterbium-doped large-core fiber laser with 633-W continuous-wave output power," Optics Letters, vol. 30, no. 9, pp. 955-957, 2005.

[6] Q. Wang, L. Zhang, C. Sun, and Q. Yu, "Multiplexed fiberoptic pressure and temperature sensor system for down-hole measurement," IEEE Sensors Journal, vol. 8, no. 11-12, Article ID 4666724, pp. 1879-1883, 2008.

[7] S. Watson, M. J. Gander, W. N. MacPherson et al., "Lasermachined fibers as Fabry-Perot pressure sensors," Applied Optics, vol. 45, no. 22, pp. 5590-5596, 2006.

[8] S. Trpkovski, S. A. Wade, S. F. Collins, and G. W. Baxter, " $\mathrm{Er}^{3+}: \mathrm{Yb}^{3+}$ doped fibre with embedded FBG for simultaneous measurement of temperature and longitudinal strain," Measurement Science and Technology, vol. 16, no. 2, pp. 488-496, 2005.

[9] J. Mandal, S. Pal, T. Sun, K. T. V. Grattan, A. T. Augousti, and S. A. Wade, "Bragg grating-based fiber-optic laser probe for temperature sensing," IEEE Photonics Technology Letters, vol. 16, no. 1, pp. 218-220, 2004.

[10] H. H. Kee, G. P. Lees, and T. P. Newson, "All-fiber system for simultaneous interrogation of distributed strain and temperature sensing by spontaneous Brillouin scattering," Optics Letters, vol. 25, no. 10, pp. 695-697, 2000.

[11] M. Niklès, L. Thévenaz, and P. A. Robert, "Simple distributed fiber sensor based on Brillouin gain spectrum analysis," Optics Letters, vol. 21, no. 10, pp. 758-760, 1996.

[12] P. M. Talaia, A. Ramos, I. Abe et al., "Plated and intact femur strains in fracture fixation using fiber Bragg gratings and strain gauges," Experimental Mechanics, vol. 47, no. 3, pp. 355-363, 2007.

[13] E. J. Seibel, R. E. Carroll, J. A. Dominitz et al., “Tethered capsule endoscopy, a low-cost and high-performance alternative 
technology for the screening of esophageal cancer and Barrett's esophagus," IEEE Transactions on Biomedical Engineering, vol. 55, no. 3, pp. 1032-1042, 2008.

[14] Y. Sasaki, S. Tanosaki, J. Suzuki et al., "Fundamental imaging properties of transillumination laser CT using optical fiber applicable to bio-medical sensing," IEEE Sensors Journal, vol. 3, no. 5, pp. 658-667, 2003.

[15] L. Quintino, A. Costa, R. Miranda, D. Yapp, V. Kumar, and C. J. Kong, "Welding with high power fiber lasers-a preliminary study," Materials and Design, vol. 28, no. 4, pp. 1231-1237, 2007.

[16] A. Polynkin, P. Polynkin, M. Mansuripur, and N. Peyghambarian, "Single-frequency fiber ring laser with $1 \mathrm{~W}$ output power at $1.5 \mu \mathrm{m}$," Optics Express, vol. 13, no. 8, pp. 3179-3184, 2005.

[17] Y. Jeong, C. Alegria, J. K. Sahu et al., "A 43-W C-band tunable narrow-linewidth erbium-ytterbium codoped large-core fiber laser," IEEE Photonics Technology Letters, vol. 16, no. 3, pp. 756-758, 2004.

[18] Z. G. Lu, F. G. Sun, G. Z. Xiao, P. Lin, and P. Zhao, "Highpower multiwavelength $\mathrm{Er}^{3+}-\mathrm{Yb}^{3+}$ codoped double-cladding fiber ring laser," IEEE Photonics Technology Letters, vol. 17, no. 9, pp. 1821-1823, 2005.

[19] X. X. Yang, L. Zhan, Q. S. Shen, and Y. X. Xia, "High-power single-longitudinal-mode fiber laser with a ring Fabry-Pérot resonator and a saturable absorber," IEEE Photonics Technology Letters, vol. 20, no. 11, pp. 879-881, 2008.

[20] P. W. Milonni and J. H. Eberly, Laser Physics, John Wiley \& Sons, New York, NY, USA, 2010.

[21] D. Kouznetsov and J. V. Moloney, "Efficiency of pump absorption in double-clad fiber amplifiers. II. Broken circular symmetry," Journal of the Optical Society of America B, vol. 19, no. 6, pp. 1259-1263, 2002.

[22] G. Das and Z. J. Chaboyer, "Single-wavelength fiber laser with $250 \mathrm{~mW}$ output power at $1.57 \mu \mathrm{m}$," Optics Express, vol. 17, no. 10, pp. 7750-7755, 2009.

[23] P. Urquhart, "Compound optical-fiber-based resonators," Journal of the Optical Society of America A, vol. 5, no. 6, pp. 803-812, 1988.

[24] S. J. Hogeveen and H. Vandestadt, "Fabry-Perot interferometers with 3 mirrors," Applied Optics, vol. 25, no. 22, pp. 41814184, 1986.

[25] H. Vandestadt and J. M. Muller, "Multimirror Fabry-Perot interferometers," Journal of the Optical Society of America A, vol. 2, no. 8, pp. 1363-1370, 1985.

[26] Y. Cheng, J. T. Kringlebotn, W. H. Loh, R. I. Laming, and D. N. Payne, "Stable single-frequency traveling-wave fiber loop laser with integral saturable-absorber-based tracking narrow-bandfilter," Optics Letters, vol. 20, no. 8, pp. 875-877, 1995.

[27] A. L. Schawlow and C. H. Townes, "Infrared and optical masers," Physical Review, vol. 112, no. 6, pp. 1940-1949, 1958.

[28] N. Y. Voo, P. Horak, M. Ibsen, and W. H. Loh, "Anomalous linewidth behavior in short-cavity single-frequency fiber lasers," IEEE Photonics Technology Letters, vol. 17, no. 3, pp. 546-548, 2005.

[29] P. Horak, N. Y. Voo, M. Ibsen, and W. H. Loh, "Pump-noiseinduced linewidth contributions in distributed feedback fiber lasers," IEEE Photonics Technology Letters, vol. 18, no. 9, pp. 998-1000, 2006.

[30] M. A. Lapointe and M. Piché, "Linewidth of high-power fiber lasers," in Proceedings of the SPIE: Photonics North, vol. 7386, pp. 73860S-1-73860S-8, 2009.

[31] V. Roy, M. Piché, F. Babin, and G. W. Schinn, "Nonlinear wave mixing in a multilongitudinal-mode erbium-doped fiber laser," Optics Express, vol. 13, no. 18, pp. 6791-6797, 2005. 

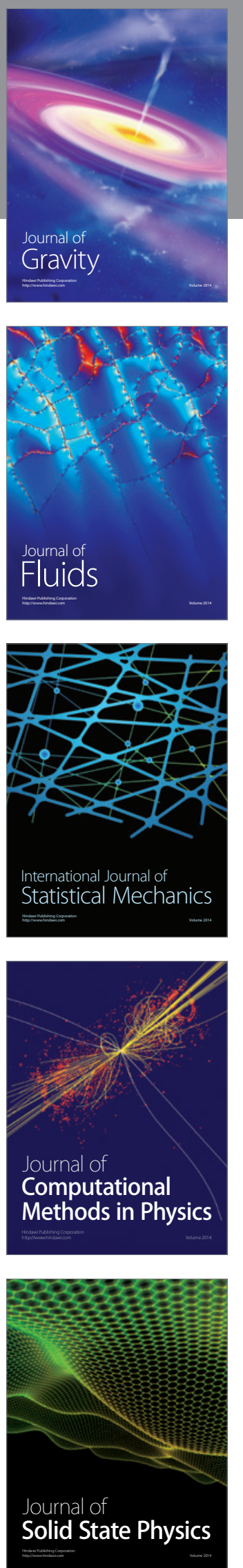

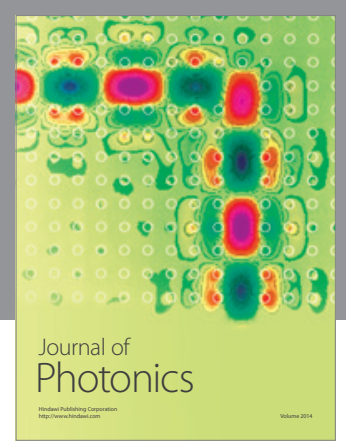

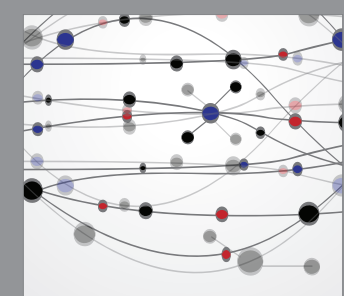

The Scientific World Journal
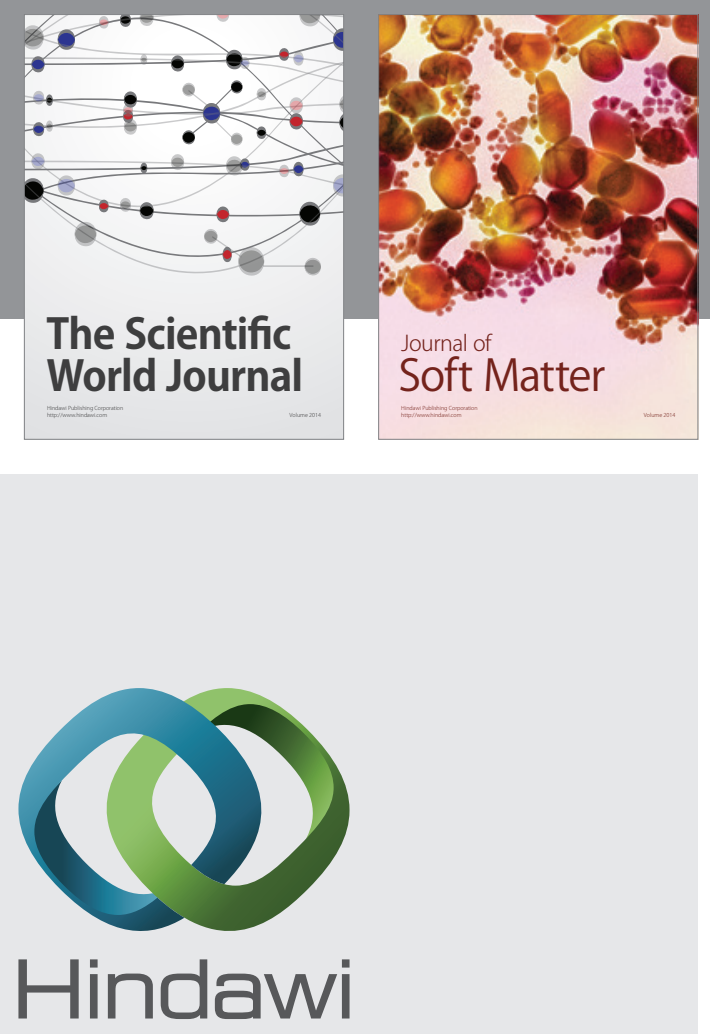

Submit your manuscripts at

http://www.hindawi.com
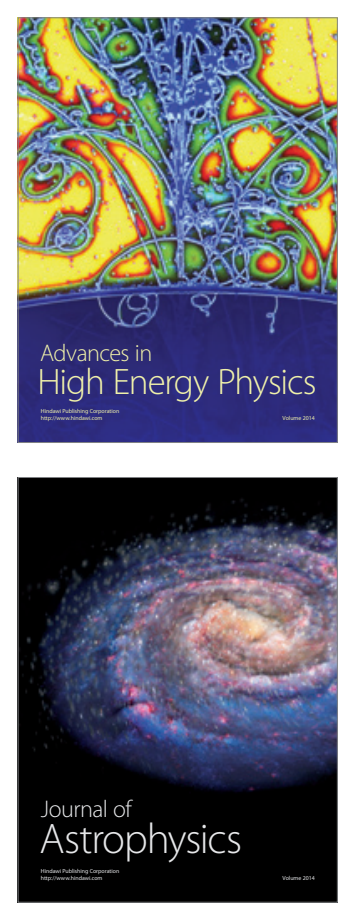
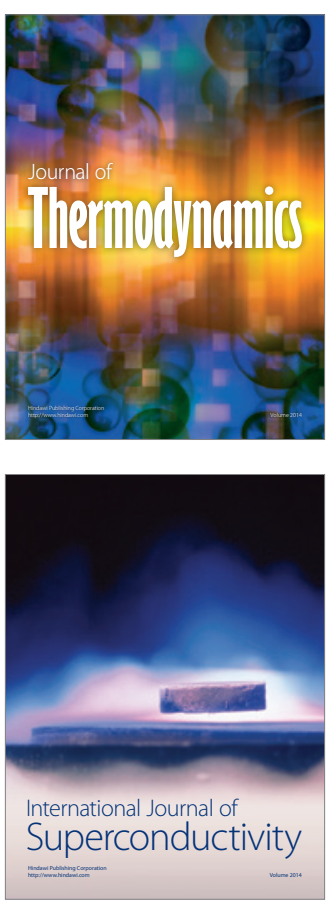
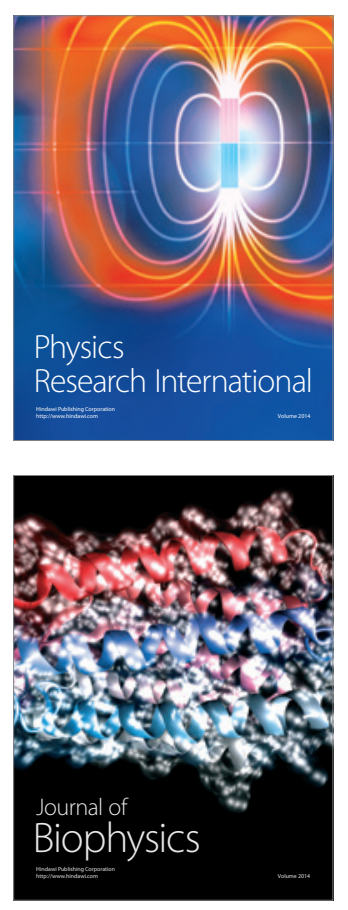
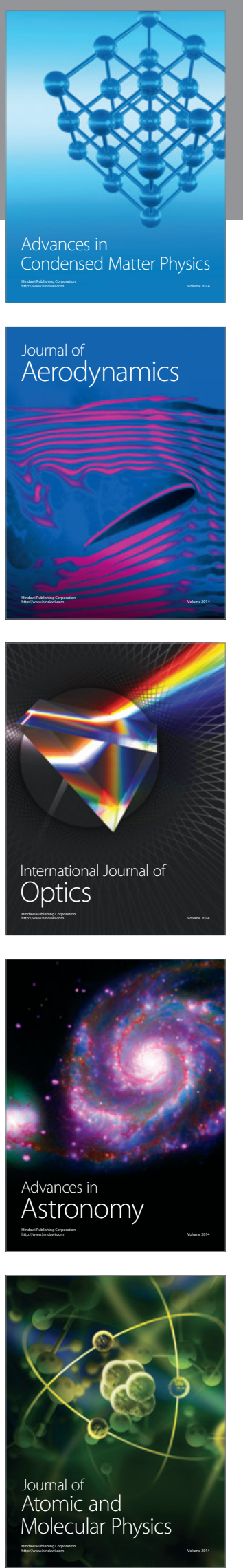